\title{
Constraints on free parameters of the simplest bilepton gauge model from the neutral kaon system mass difference
}

\author{
F. Pisano \\ Departamento de Física, Universidade Federal do Paraná, 81531-990 Curitiba, PR, Brazil \\ J. A. Silva-Sobrinho \\ Instituto de Física Teórica, Universidade Estadual Paulista, Rua Pamplona 145, 01405-900 São Paulo, SP, Brazil \\ M. D. Tonasse* \\ Instituto de Física, Universidade do Estado do Rio de Janeiro, Rua São Francisco Xavier 524, 20550-013 Rio de Janeiro, RJ, Brazil
}

(Received 5 February 1998; published 29 July 1998)

\begin{abstract}
We consider the contributions of the exotic quarks and gauge bosons to the mass difference between the short- and the long-lived neutral kaon states in the $\mathrm{SU}(3)_{C} \times \mathrm{SU}(3)_{L} \times \mathrm{U}(1)_{N}$ model. The lower bound $M_{Z^{\prime}} \sim 14$ $\mathrm{TeV}$ is obtained for the extra neutral gauge boson $Z^{\prime 0}$. Ranges for values of one of the exotic quark masses and quark mixing parameters are also presented.
\end{abstract}

[S0556-2821(98)05215-1]

PACS number(s): 12.60.-i, 12.15.Ff, 14.70.Pw

The $\Delta m=m_{K_{L}}-m_{K_{S}}$ mass difference between the longand the short-lived kaon states was successfully used in a two-generation standard model to predict the charmed quark mass [1]. In the following years several authors studied the $K^{0}-\bar{K}^{0}$ system in order to find constraints on the parameters of new gauge theories such as gauge and scalar boson masses and mixing angles (see, for example, Refs. [2-4]). The idea behind this is that, since the $c$ quark mass was predicted with good precision, a possible new contribution to $\Delta m$ must be smaller than the result obtained from the two generations.

In this paper we go back to this subject in order to constrain the new neutral gauge boson mass and quark mixing parameters imposed by the $\mathrm{SU}(3)_{C} \times \mathrm{SU}(3)_{L} \times \mathrm{U}(1)_{N}(3-3-1)$ model [5-7]. The 3-3-1 model is a gauge theory based on the $\mathrm{SU}(3)_{C} \otimes \mathrm{SU}(3)_{L} \otimes \mathrm{U}(1)_{N}$ semisimple symmetry group. It has the interesting feature that the anomaly cancellation does not happen within each generation, as in the standard model, but only when the three generations are considered together. Thus, the number of families must be a multiple of the color degrees of freedom and, as a consequence, the 3-3-1 model suggests a route towards the solution of the flavor question [5-8].

Let us summarize the most relevant points of the model. In the minimal particle content of Ref. [5] the left-handed quark fields transform under the $\mathrm{SU}(3)_{L}$ group as the triplets

$$
Q_{1 L}=\left(\begin{array}{c}
u_{\varphi} \\
d_{1 \theta} \\
J_{1}
\end{array}\right)_{L} \sim\left(\mathbf{3}, \frac{2}{3}\right), \quad Q_{\alpha L}=\left(\begin{array}{c}
J_{\alpha \phi} \\
u_{\alpha \varphi} \\
d_{\alpha \theta}
\end{array}\right)_{L} \sim\left(3^{*},-\frac{1}{3}\right)
$$

*Email address: Tonasse@vax.fis.uerj.br $(\alpha=2,3)$, where $2 / 3$ and $-1 / 3$ are the $\mathrm{U}(1)_{N}$ charges. Each left-handed quark field has its right-handed counterpart transforming as a singlet of the $\mathrm{SU}(3)_{L}$ group. In order to avoid anomalies one of the quark families must transform in a different way with respect to the two others. In Ref. [6] the singled family is the third one, but this is not relevant here. The exotic quark $J_{1}$ carries $5 / 3$ units of electric charge while $J_{2}$ and $J_{3}$ carry $-4 / 3$ each. In the gauge sector the singlecharged $\left(V^{ \pm}\right)$and the double-charged $\left(U^{ \pm \pm}\right)$vector bileptons [9], together with a new neutral gauge boson $Z^{\prime 0}$, complete the particle spectrum with the charged $W^{ \pm}$and the neutral $Z^{0}$ standard gauge bosons. At low energy the 3-3-1 model recovers the standard phenomenology $[7,10]$.

An important property is that the bileptons can have a low energy scale. Low energy data constrain the vector bilepton masses to $M_{X}>230 \mathrm{GeV}\left(X \equiv V^{+}, U^{++}\right)$[11]. Some authors have used the running of the coupling constant to impose upper bounds on 3-3-1 gauge boson masses $[12,13]$. However, this procedure involves an arbitrary normalization of $N[6,7]$. Usually this is done like in the standard model, although it is not mandatory. Recently these upper bounds were reexamined and it was found that the $M_{Z^{\prime}}$ mass does not have upper bound, differently from previous calculations, while $M_{X}<3.5 \mathrm{TeV}$ [14].

The $\Delta m$ mass difference has already been studied in the context of the 3-3-1 model at the tree level (Fig. 1a) in order to put a lower bound on the $M_{Z^{\prime}}$ mass [15] and on the mixing parameters [13], in the last case, taking into account an upper bound on $M_{Z^{\prime}}$. Here we apply the experimental lower limits and these reexamined upper bounds on the $M_{X}$ gauge boson masses in order to obtain the impositions of $\Delta m$ upon some of the 3-3-1 free parameters. Since the bileptons couple exotic to ordinary quarks, leading to additional contributions to the box diagram for the $K^{0}-\bar{K}^{0}$ transition (Fig. 1b), we combine the tree level with the box contribution.

The charged current interactions for the quarks are given in Ref. [5] and we can rewrite them as 


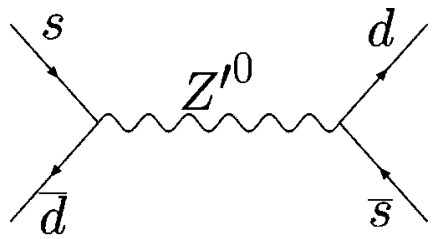

(a)

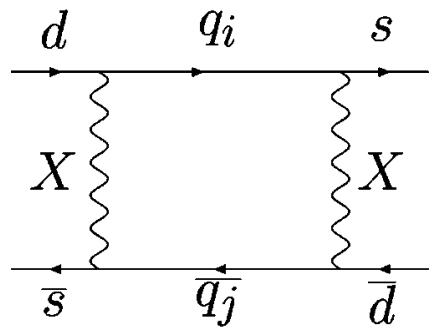

(b)

FIG. 1. $K^{0}-\bar{K}^{0}$ transition originating the $\Delta m=m_{K_{L}}-m_{K_{S}}$ mass difference at the tree level (a) and the box diagram (b) in the 3-3-1 model. The $q$ 's are all quarks coupled by $X$ gauge bosons to $d$ and $s$

$$
\begin{aligned}
\mathcal{L}= & -\frac{g}{2 \sqrt{2}}\left[\bar{U} \gamma^{\mu}\left(1-\gamma_{5}\right) V_{\mathrm{CKM}} D W_{\mu}^{+}+\bar{U} \gamma^{\mu}\left(1-\gamma_{5}\right) \zeta \mathcal{J} V_{\mu}\right. \\
& \left.+\bar{D} \gamma^{\mu}\left(1-\gamma_{5}\right) \xi \mathcal{J} U_{\mu}\right]+ \text { H.c. },
\end{aligned}
$$

where

$$
U=\left(\begin{array}{c}
u \\
c \\
t
\end{array}\right), \quad D=\left(\begin{array}{c}
d \\
s \\
b
\end{array}\right), \quad \mathcal{V}_{\mu}=\left(\begin{array}{c}
V_{\mu}^{+} \\
U_{\mu}^{-} \\
U_{\mu}^{--}
\end{array}\right), \quad \mathcal{U}_{\mu}=\left(\begin{array}{c}
U_{\mu}^{--} \\
V_{\mu}^{+} \\
V_{\mu}^{+}
\end{array}\right) \text {, }
$$

and $\mathcal{J}=\operatorname{diag}\left(\begin{array}{lll}J_{1} & J_{2} & J_{3}\end{array}\right)$. The $V_{\mathrm{CKM}}$ is the usual CabibboKobayashi-Maskawa mixing matrix and $\zeta$ and $\xi$ are mixing matrices containing the new unknown parameters due to the presence of the exotic quarks. Here, unlike Eq. (1), we are working with the mass eigenstates.

We are using the unitary gauge. The box diagram for the $K^{0}-\bar{K}^{0}$ transition is represented in Fig. 1b, where, in the standard model, $q_{i}=U_{i}(i=1,2,3)$ and $X$ is the $W^{-}$boson. In the 3-3-1 model, besides these, we have new contributions: for $i=1, q_{1}=J_{1}$ and $X=U^{--}$, while for $i=\alpha=2,3, q_{\alpha}=J_{\alpha}$ with $X=V^{-}$. We do not consider here contributions of the scalar bileptons. This would be very complicated because of the proliferation of unknown parameters as mixing angles and scalar boson masses [16]. However, because of the elu- sivity of the scalar particles, we expect that the new contributions of the 3-3-1 model will be dominated by the gauge bosons.

Our calculation is standard [1]. Neglecting long-distance terms, we define $\mathcal{L}_{X}$-an effective Lagrangian corresponding to the $X$ gauge boson exchange in the box diagram-as being the free quark amplitude $A_{X}(d \bar{s} \rightarrow \bar{d} s)$ and we evaluate the matrix element $\left\langle\bar{K}^{0}\left|-\mathcal{L}_{X}\right| K^{0}\right\rangle$. According to the MIT bag model we can write [4]

$$
A_{X}=-0.7\left(\frac{G_{F} M_{W}^{2}}{\pi M_{X}}\right)^{2} \mathcal{O}_{s d} \sum_{i, j} \Gamma_{i} \Gamma_{j} \frac{f\left(a_{q_{i}}^{X}\right)-f\left(a_{q_{j}}^{X}\right)}{a_{q_{i}}^{X}-a_{q_{j}}^{X}}
$$

where the $\Gamma_{i}$ are mixing parameters given in the standard model by $V_{i s}^{*} V_{i d}$ and in the 3-3-1 model by $\xi_{i s}^{*} \xi_{i d}$. From the momentum integration we have

$$
f(x)=\frac{1}{1-x}\left(1+\frac{x^{2} \ln x}{1-x}\right)
$$

In Eq. (4), $a_{q_{i}}^{X}=\left(m_{q_{i}} / M_{X}\right)^{2}$ is the square ratio of the $m_{q_{i}}$ quark and the $M_{X}$ gauge boson masses. We have defined $\mathcal{O}_{s d} \equiv\left[\overline{v_{s}} \gamma_{\mu}\left(1-\gamma_{5}\right) u_{d}\right]^{2}$. The sum in Eq. (4) runs over all quarks coupled by the $X$ gauge boson. The $\Delta m_{X}$ mass difference due to the $X$ gauge boson contribution is given by

$$
\Delta m_{X}=-2 \operatorname{Re}\left\langle\bar{K}^{0}\left|\mathcal{L}_{X}\right| K^{0}\right\rangle,
$$

with $\mathcal{L}_{X} \equiv A_{X}$. Taking into account the relation

$$
\left\langle\bar{K}^{0}\left|\mathcal{O}_{s d}\right| K^{0}\right\rangle=\frac{4}{3} f_{K}^{2} m_{K}
$$

where $m_{K}=498 \mathrm{MeV}$ and $f_{K}=161 \mathrm{MeV}$ are the kaon meson mass and its decay constant, respectively, the total mass difference is

$$
\Delta m=\frac{8 \eta m_{K}}{3}\left(\frac{f_{K} M_{W}}{s_{W} M_{X}}\right)^{2} \sum_{i, j} \Gamma_{i} \Gamma_{j} \frac{f\left(a_{q_{i}}^{X}\right)-f\left(a_{q_{j}}^{X}\right)}{a_{q_{i}}^{X}-a_{q_{j}}^{X}},
$$

where $s_{W}=\sin \theta_{W}, \theta_{W}$ is the Weinberg weak mixing angle, and we have included the leading order QCD correction factor $\eta=0.55[13,17]$.

Despite the uncertainties undergone by the evaluation of the effective Lagrangian $\mathcal{L}_{X}$, the application of this method to well-known processes tells us that the procedure is reliable [3].

In order to get physical results from Eq. (8) we perform some hypotheses on the free parameters. First, we notice that the standard model does not give the whole $\Delta m$ mass difference. Considering only two quark generations we find $\Delta m_{2}$ $=2 \times 10^{-15} \mathrm{GeV}$, while the experimental value is $\Delta m_{\text {expt }}$ $=3.491 \times 10^{-15} \mathrm{GeV}$ [18]. The top quark contribution is negligible because of the smallness of the corresponding 


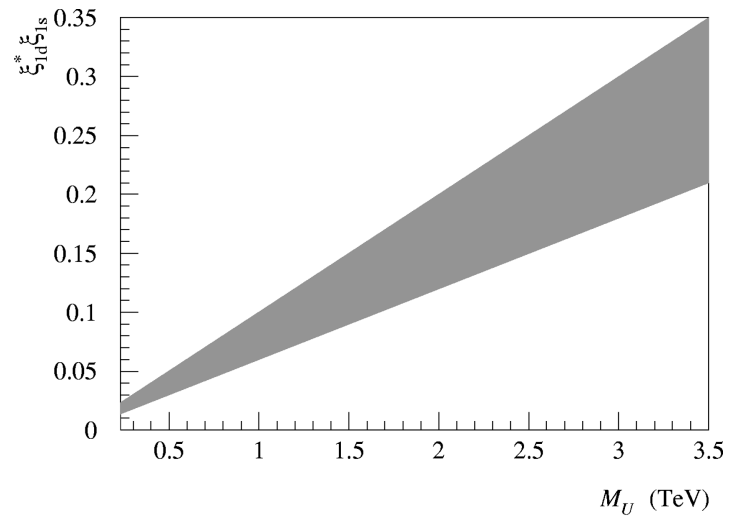

FIG. 2. Bounds on quark mixing parameters from the $m_{K_{L}}$ $-m_{K_{S}}$ mass difference as a function of the double-charged bilepton mass. The dark region represents the allowed values for $\xi_{1 d}^{*} \xi_{1 s}$.

mixing parameters. Thus, if the 3-3-1 model is realized in nature, we can expect that its pure contribution to $\Delta m$ is about $10^{-15} \mathrm{GeV}$.

Let us examine individual 3-3-1 contributions under the conservative hypothesis in which each one of them gives the total assumed 3-3-1 counterpart to $\Delta m$, i.e., $10^{-15} \mathrm{GeV}$. We begin analyzing the contribution of the $U$ bilepton and the $J_{1}$ quark. For convenience we assume for the $\xi$ mixing matrix the same parametrization advocated by the Particle Data Group for $V_{\mathrm{CKM}}$ [18]. In this case, since we choose all the mixing angles in the first quadrant, the parameter $\xi_{1 d}^{*} \xi_{1 s}$ is positive. We do not consider here $C P$ violating phases in the mixing matrices. In Fig. 2 we plot this mixing parameter as function of $M_{U}$, according to Eq. (8), where we are using the bounds $M_{U}>230 \mathrm{GeV}$ [11] and $M_{U}<3500 \mathrm{GeV}$ [14]. Hence, we can see that $0.014 \lesssim \xi_{1 d}^{*} \xi_{1 s} \lesssim 0.35$. Physically this range for the mixing angles implies $m_{J_{1}} \lesssim M_{U}$. In order to apply the lower limit on the mixing parameter for obtaining a lower bound for $M_{Z^{\prime}}$, we use the result of Ref. [15] for the
$Z^{\prime}$ contribution to $\Delta m$, but taking into account that we are assuming that the maximal contribution to $\Delta m$ is $\sim 10^{-15}$ $\mathrm{GeV}$ (not the whole experimental value). We introduce also the leading order QCD correction factor $\eta=0.55$. This leads to $M_{Z^{\prime}} \sim 1.03 \times 10^{3}\left[\operatorname{Re}\left(V_{L 11}^{D *} V_{L 12}^{D}\right)\right]^{1 / 2} \mathrm{TeV}$, where $V_{L}^{D}$, the mixing matrix relating the symmetry left-handed quark states carrying $-1 / 3$ units of electric charge $\left(D_{L}^{\prime}\right)$ with the physical ones $\left(D_{L}\right)$, is defined by $D_{L}^{\prime}=V_{L}^{D} D_{L}$. Since only the $J_{1}$ quark carries $5 / 3$ units of electric charge, does it not mix. Therefore, the mixing parameter $V_{L 11}^{D *} V_{L 12}^{D}$ is the same as $\xi_{1 s}^{*} \xi_{1 d}$, whose lower bound gets $M_{Z}^{\prime} \sim 14.4 \mathrm{TeV}$.

A more rigorous analysis, considering the contributions of diagrams exchanging the single-charged $V$ bilepton and $J_{2}$ and $J_{3}$ exotic quarks, is complicated since these two quarks can mix and the sign of the mixing parameters is not defined according to our parametrization of the mixing matrices and the choice of the mixing angles. However, it is not expected to make an appreciable difference in the results.

In this Brief Report we have studied the implications of the $\Delta m$ mass difference on free parameters of the 3-3-1 model. However, differently from previous calculations $[13,15]$, here we are taking into account the contribution of the box diagram exchanging the double-charged $U^{--}$bilepton. Our results differ from the previous ones because we have combined the analysis for the tree level and the box diagram. Therefore, if the 3-3-1 Higgs contribution to $\Delta m$ is not important, we can assume the value we have estimated for $M_{Z^{\prime}}$ as an approximate lower bound (i.e., $M_{Z^{\prime}} \gtrsim 14 \mathrm{TeV}$ ). We stress that this bound does not depend on the aforementioned arbitrary normalization of $N$. The crucial parameter for this value is the experimental lower bound on the mass of the $U^{--}$bilepton gauge boson.

We would like to thank Dr. M. C. Tijero for reading the manuscript and the Instituto de Física Teórica, UNESP, for use of its facilities. The work of M. D. T. was supported by the Fundação de Amparo à Pesquisa no Estado do Rio de Janeiro (proc. No. E-26/150.338/97).
[1] M. K. Gaillard and B. W. Lee, Phys. Rev. D 10, 897 (1974).

[2] V. Barger, W. F. Long, and S. Pakvasa, Phys. Rev. Lett. 42, 1585 (1979); L. F. Abbott, P. Sikivie, and M. B. Wise, Phys. Rev. D 21, 1393 (1980); V. Barger, J. L. Hewett, and R. Phillips, ibid. 41, 3421 (1990); Y. Grossman, Nucl. Phys. B426, 355 (1994).

[3] G. Beall, M. Bander, and A. Soni, Phys. Rev. Lett. 48, 848 (1982).

[4] R. E. Shrock, S. B. Treiman, and L.-L. Wang, Phys. Rev. Lett. 42, 1589 (1979); A. Buras, ibid. 46, 1354 (1981).

[5] F. Pisano and V. Pleitez, Phys. Rev. D 46, 410 (1992); R. Foot, O. F. Hernandez, F. Pisano, and V. Pleitez, ibid. 47, 4158 (1993).

[6] P. H. Frampton, Phys. Rev. Lett. 69, 2889 (1992).

[7] A review of the 3-3-1 model is given by F. Pisano, V. Pleitez, and M. D. Tonasse, "Flavor chiral extensions for the standard model," Report No. IFT-P.043/97.

[8] F. Pisano, Mod. Phys. Lett. A 11, 2639 (1996).
[9] For a recent review on bileptons see F. Cuypers and S. Davidson, Eur. Phys. J. C2, 503 (1998).

[10] J. T. Liu and D. Ng, Z. Phys. C 62, 693 (1994); D. Ng, Phys. Rev. D 49, 4805 (1994).

[11] E. D. Carlson and P. H. Frampton, Phys. Lett. B 283, 123 (1992).

[12] K. Sasaki, Phys. Lett. B 383, 297 (1993).

[13] J. T. Liu, Phys. Rev. D 50, 542 (1994).

[14] P. Jain and S. D. Joglekar, Phys. Lett. B 407, 151 (1997).

[15] D. Gómez Dumm, F. Pisano, and V. Pleitez, Mod. Phys. Lett. A 9, 1609 (1994). Notice that the unitarity condition of the quark mixing matrix $V_{L}^{D}$ was used to obtain the $\overline{d_{L}} s_{L} Z^{\prime}$ coupling appearing in Eq. (14a) in this reference.

[16] The scalar sector of the 3-3-1 models is treated by M. D. Tonasse, Phys. Lett. B 381, 191 (1996) (see also Ref. [7]).

[17] F. J. Gilman and M. B. Wise, Phys. Lett. 93B, 129 (1980).

[18] Particle Data Group, R. M. Barnett et al., Phys. Rev. D 54, 1 (1996). 